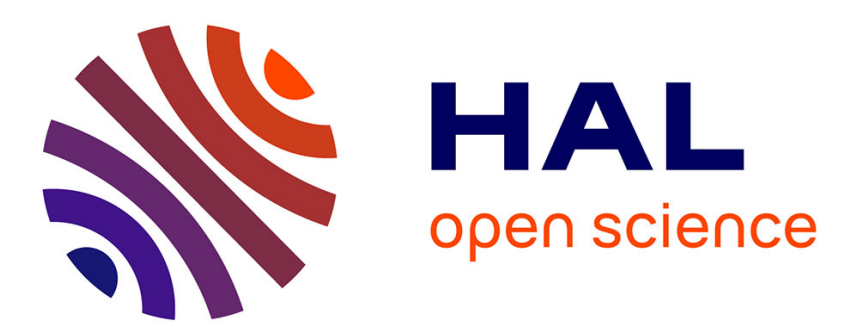

\title{
Les apports du cinéma à une (géo-)graphie des frontières mobiles et des migrations frontalières
}

\author{
Sarah Mekdjian
}

\section{To cite this version:}

Sarah Mekdjian. Les apports du cinéma à une (géo-)graphie des frontières mobiles et des migrations frontalières. Annales de géographie, 2014, Géographie et cinéma, 1-2, pp.784-804. 10.3917/ag.695.0784 . halshs-01242522

\section{HAL Id: halshs-01242522 \\ https://shs.hal.science/halshs-01242522}

Submitted on 14 Dec 2015

HAL is a multi-disciplinary open access archive for the deposit and dissemination of scientific research documents, whether they are published or not. The documents may come from teaching and research institutions in France or abroad, or from public or private research centers.
L'archive ouverte pluridisciplinaire HAL, est destinée au dépôt et à la diffusion de documents scientifiques de niveau recherche, publiés ou non, émanant des établissements d'enseignement et de recherche français ou étrangers, des laboratoires publics ou privés. 


\section{Sarah Mekdjian}

Département de géographie sociale, Université Pierre Mendès France,

\section{Grenoble II}

Texte à paraître-Annales de Géographie, numéro spécial «Géographie et cinéma », coordination Jean-François Staszak, Université de Genève

Les apports du cinéma à une (géo-)graphie des frontières mobiles et des déplacements frontaliers

\section{Résumé}

Les frontières sont irréductibles à des tracés géopolitiques linéaires. Leurs fonctions de barrière ou d'interface se diffusent dans l'espace et dans le temps, en fonction des politiques étatiques, de l'intensité et de la diversité des flux migratoires, qui s'y déploient. Nous proposons d'appréhender les frontières depuis les déplacements et les migrations qu'elles génèrent, c'està-dire depuis leur dynamique. Art du temps et du mouvement, le cinéma peut-il contribuer aux réflexions géographiques sur les déplacements frontaliers et la frontière mobile, en tant qu'étendue instable, constituée «chemin faisant»? En quoi le cinéma de fiction peut-il participer à l'élaboration d'un projet cognitif géographique ? Les caractéristiques des images cinématographiques ouvrent-elles des perspectives pour la figuration des frontières mobiles en géographie?

Mots-clés : cinéma, fiction, frontière, géographie, cartographie, migration, Amérique

Cinematic maps of mobile borders and cross-borders migrations
Abstract
Borders can not be reduced to more or less continuous lines, in a surface- based and timeless vision of space. Border regions attract dense networks of 
migrations. Documenting and mapping the mobility of the border and within the border region is a ongoing challenge in geography. As an art of « movement-image », according to Deleuze's theory, could cinema help produce a mobile geography and cartography of borders? This paper explores the cartographic dimensions of cinema and its potential to suggest new maps of cross-border migrations. Frames, shots and montage, as creations of innovative time/space configurations, will be analyzed in two films showing the US/Mexico border : Sin Nombre, written and directed by Cary Fukunaga in 2009 and No Country For Old Men, written and directed by Joe and Ethan Coen in 2007, after Cormac McCarthy's eponymous novel.

Key words : cinema, fiction, border, geography, cartography, migration, North America 
Nos bistros et nos avenues de métropoles, nos bureaux et chambres meublées, nos gares et nos usines paraissaient devoir nous enfermer sans espoir d'y échapper jamais. Vint le film, qui fit sauter ce monde-prison par la dynamite des dixièmes de seconde, si bien que désormais, au milieu de ses ruines et débris au loin projetés, nous faisons insoucieusement d'aventureux voyages. Sous la prise de vue à gros plan s'étend l'espace, sous le temps de pose se développe le mouvement. Walter Benjamin, « L'œuvre d'art à l'ère de sa reproductibilité technique », première version (1935), trad. R. Rochlitz , in Euvres, t.III, Paris Gallimard « Folio essais », 2000

Les frontières ne sont pas réductibles à des tracés linéaires continus. Leurs fonctions de barrière ou d'interface évoluent en fonction de l'intensité et de la diversité des flux migratoires, qu'elles génèrent. Les modes de surveillance des frontières étatiques sont en voie d'externalisation ${ }^{1}$, principalement depuis les années 1990 (Audebert, Robin, 2009) et tentent de s'adapter à la géographie dynamique des trajectoires migratoires. Du point de vue des migrants illégaux, les espaces frontaliers, en opposition à la ligne, correspondent bien souvent à tout l'espace parcouru pendant le voyage ; les difficultés matérielles, les menaces d'arrestation, sont autant de barrières qui se déploient tout au long du trajet. A partir d'une analyse des processus d'ouverture et de fermeture des frontières, en relation avec les mobilités mondiales, Anne-Laure Amilhat-Szary et Frédéric Giraut définissent la notion de «frontière mobile» (Amilhat-Szary, 2012; Amilhat-Szary, Giraut, 2011) : « les fonctions frontalières tendent à dépasser la localisation sur les limites établies des aires de souveraineté nationale, pour être repoussées, projetées, multipliées ou diffusées dans l'espace » (Amilhat-Szary, Giraut, 2011). La frontière mobile n'équivaut donc pas au front ou à la marche ; il ne s'agit pas d'une limite repoussée par des activités de conquête, mais bien d'un objet dont les fonctions sont diffusées et

1 Le terme «externalisation », issu du vocabulaire économique, désigne, dans le registre géopolitique de l'étude des frontières, «la volonté des pays européens comme celle des États-Unis de conférer aux pays du Sud une responsabilité croissante de la gestion et du contrôle des frontières des pays du Nord» (Audebert, Robin, 2009, p. 35). Cédric Audebert et Nelly Robin montrent que si la définition du terme en géopolitique n'est pas clairement délimitée, 1 ' «externalisation » peut «aider à mieux cerner les implications des politiques en question sur la dynamique des flux et notamment la diversification des stratégies spatiales » (ibid.). 
transformées dans l'espace et dans le temps. La variabilité spatio-temporelle des frontières, en relation avec la géographie des migrations, emprunte à la pensée de l'espace mobile développée par Denis Retaillé, qui explique que «le mouvement étant permanent, il est de plus en plus difficile de croire à des limites du type des frontières nationales. [...] la limite à deux bords que constitue la frontière se lézarde et [...] les limites [...] se diluent dans [1']espace » (Retaillé, 2011).

Les analyses sur les dimensions dynamiques de l'espace ont été renforcées par les recherches en géomatique, qui ont contribué à introduire le mouvement et le temps dans le registre des représentations spatiales. Ainsi, les cartographies dynamiques, animées, interactives ${ }^{2}$, mais aussi les recherches sur les graphes ${ }^{3}$, ont transformé les modes de visualisation des flux (Cheylan, 2007 ; Kaddouri, 2008 ) et des trajectoires spatio-temporelles (Équipe Mobydic, 2010). Les cartes animées, alternatives «à la carte statique [...] permettent une meilleure représentation de la dynamique » (Kaddouri, 2008, p. 2), et notamment des processus de discontinuités spatiales et temporelles générés par les frontières, principalement étudiées depuis leurs effets de barrière.

Dans un autre registre de production cartographique, associant cartographes, artistes et militants, la «contre-cartographie» ou « countermapping » s'est saisie des questions frontalières et migratoires. Les cartes produites, très hétérogènes tant sur le fond que sur la forme, mêlent le plus souvent une visée cognitive, esthétique et critique. Il s'agit à la fois de « rendre visible l'invisible » (Gresh, Rekacewicz, 2012) et de dénoncer les modes politiques dominants de représentation d'objets géographiques, par exemple les représentations sécuritaires des frontières et des migrations ${ }^{4}$.

2 La carte animée des migrations de footballeurs réalisée par Raffaele Poli par exemple associe la spatialisation des trajectoires des individus à une chronologie (Poli, 2007).

3 Le groupe de recherche FMR, Flux, Matrices, Réseaux, développe des outils et des analyses dans le domaine de la visualisation des flux et des réseaux (http://groupefmr.hypotheses.org/), tandis que le groupe de recherche CartoMouv', cartographie du changement, cartographies en mouvement, travaille sur «la représentation graphique des temporalités et des dynamiques spatiales et sur les nouvelles techniques permettant davantage d'interactivité et d'animation dans la production des cartes » (http://www.cartomouv.parisgeo.cnrs.fr/).

4 La carte du nombre de morts aux frontières de l'Union Européenne (Migreurop, Clochard, 2009) contribue à spatialiser et rendre visible des données statistiques, dont la représentation était jusqu'alors inexistante. La carte des migrations à travers le Détroit 
La question de la visualisation des processus spatio-temporels, et les essais de croisement des approches scientifiques et artistiques, amènent à s'intéresser au cinéma, pour élaborer une géographie de la frontière mobile. Le cinéma, «art générateur d'espaces » (Castro, 2011, p. 8) et « art du mouvement et du temps » (Montebello, 2008, p. 9), entretient des relations étroites avec la géographie et la cartographie. Tom Conley (2007), qui étudie ces relations à partir de nombreuses analyses filmiques, insiste sur la posture du spectateur au cinéma, comparée à celle de l'observateur d'une carte. Ses travaux portent aussi spécifiquement sur l'apparition de cartes à l'écran. Plus récemment, Teresa Castro (2011) démontre l'existence d'une « raison cartographique des images » et explique que «le cinéma et, au-delà de celui-ci, le monde des images en général sont traversés par une pensée de l'espace qui trouve dans la carte géographique son modèle théorique $\gg$ (Castro, 2011, p. 9). Du point de vue des apports des images cinématographiques à la cartographie, Sébastien Caquard (2009) retrace l'origine de la cartographie animée et 3D dans l'histoire du cinéma.

A la suite de ces travaux et à partir de l'analyse de deux films de fiction, Sin Nombre de Cary Fukunaga (2009) et No Country For Old Men de Ethan et Joel Cohen (2007), nous proposons de montrer comment les images cinématographiques, qui procèdent par un double découpage spatial et temporel : le cadre et le plan, peuvent contribuer aux réflexions géographiques et cartographiques sur la frontière mobile. Ces œuvres seront abordées selon deux registres : à la fois en tant que sources, susceptibles d'être mobilisées dans un projet cognitif géographique, et en tant qu'outils de visualisation de la frontière, à confronter avec les modèles cartographiques, développés en géographie. Ces deux films de fiction mettent en scène, à partir d'esthétiques distinctes, des étendues frontalières, perçues depuis le point de vue de personnages en fuite. Sin Nombre, qui signifie «Sans Nom », est le premier long-métrage réalisé en 2009 par Cary Fukunaga ${ }^{5}$. Tourné au Mexique et en espagnol, le film met en scène le voyage d'une

de Gibraltar produite par le collectif Hackitectura (collectif composé d'artistes, d'architectes, de militants) en 2006, montre les dispositifs de surveillance externalisés de l'Union Européenne, en associant une carte topographique statique à des photographies, des textes et des graphiques.

5 Cary Fukunaga a reçu, pour ce film, le prix du meilleur réalisateur à l'édition 2009 du Festival Sundance. 
jeune femme et de sa famille vers les États-Unis et la fuite croisée d'un membre de la Mara Salavatrucha à travers le Mexique. No Country For Old Men, long-métrage en anglais, a été réalisé par Ethan et Joel Cohen ${ }^{6}$ en 2007, à partir d'une adaptation du roman éponyme de Cormac McCarthy (2005). Le film montre la fuite de Llewelyn Moss à travers l'étendue frontalière du sud des États-Unis, en retravaillant les genres du western et du road movie.

Dans une première partie, nous analyserons la portée cognitive des films de fiction, dans la perspective de la géographie des frontières. Si les films documentaires référentiels sont largement mobilisés pour décrire le monde, qu'apportent les films de fiction au projet cognitif de la géographie ?

Dans la deuxième partie, nous verrons en quoi les images cinématographiques de la frontière empruntent à la cartographie et comment elles contribuent à ouvrir des pistes renouvelées pour la géographie et la cartographie des traversées frontalières.

Enfin, à partir d'une analyse de l'instabilité constitutive des images cinématographiques, nous envisagerons la possibilité d'élaborer des cartographies évanescentes de la frontière, objet dynamique et instable, qui se construit « chemin faisant ».

1-Les images cinématographiques des frontières, sources de connaissances géographiques?

L'analyse d'images cinématographiques, et plus précisément du cinéma de fiction, peut-elle s'inscrire dans la démarche cognitive de la géographie ? Si les documentaires sont souvent mobilisés comme descriptions du monde, quel est le statut de la fiction cinématographique dans l'élaboration d'une géographie de la frontière ?

-Le cinéma pour décrire et figurer le monde

Les géographes produisent de plus en plus de films ${ }^{7}$, utilisés comme

6 Le film a reçu quatre oscars en 2008: meilleur film, meilleur réalisateur, meilleur scénario adapté, meilleur second rôle masculin.

7 Le parcours universitaire intitulé «Images documentaires et géographie » au sein de 
outils d'étude de terrain et comme formes de visualisation géographique (Chenet, Simoes, Laurent, 2011). En plus de contribuer aux analyses critiques sur la réflexivité et les conditions de constitution des savoirs géographiques, le film documentaire permettrait de décrire le monde. Teresa Castro montre ainsi que les images cinématographiques peuvent servir un régime de visibilité dit descriptif, dont « l'objectif est de construire un point de vue sur la réalité et, parfois, un socle commun de savoirs » (Castro, 2011, p. 235). Cette démarche descriptive est comparable à ce que l'auteure appelle la «rationalité cartographique », qui procède d'un processus de figuration $^{8}$ ordonnée et synthétique du monde (Besse, 2008). L'acte de description, cinématographique et cartographique, «relève d'une construction de l'imagination », rappelle Teresa Castro, «car décrire, au cinéma comme ailleurs, implique une prise de position sur le réel, traduite par le choix et l'agencement d'éléments sous une certaine forme. Cet agencement $[\ldots]$ se distingue en général par des intentions précises. Parmi ces dernières se comptent souvent la formation d'une connaissance ou d'un savoir » (Castro, 2011, p. 215). Les intentions de nombreux films documentaires peuvent ainsi tout à fait s'associer au projet cognitif de la géographie. La description, mais aussi le diagramme, sont deux modes de visibilité cinématographique, qui empruntent à la «rationalité cartographique ». Le diagramme, à la différence de la description illustrative, «construit des liens et des corrélations entre des éléments discontinus d'un ensemble »(Castro, 2011, p. 228). Le montage met en relation des plans et des cadres discontinus, faisant ainsi émerger « un nouveau plan de réalité » (Besse, 2001, p. 140), à la manière d'une carte. Car toute carte comme tout film documentaire sont bien plus qu'une reproduction du monde, ils « instaure[nt] un monde autant qu'[ils] le révèle[nt] » (Besse, 2010, p. 9).

Ceci dit, ni la carte ni le cinéma n'ont nécessairement pour intention

l'UFR de géographie à l'Université de Paris I, propose une formation de l'année de L2 jusqu'au Master 1 à la production et à l'analyse $\mathrm{d}^{\prime}$ images documentaires : film documentaire, poster scientifique, graphique, carte.

8 Jean-Marc Besse préfère parler de «figuration » plutôt que de «représentation » pour désigner le «pouvoir synthétique de la carte» (Besse, 2008) : plutôt que de représenter, dans le sens de présenter à nouveau, une réalité préexistante, la carte aurait un pouvoir de «figurer » un territoire de référence, c'est-à-dire « de faire voir un objet auquel nous ne pouvons avoir un accès que dans la carte, et pas autrement » (ibid.). 
de figurer le monde référentiel. Le cinéma, comme certaines cartes, sont aussi fictionnels, créatifs d'univers où « la question de la référentialité ne compte pas » (Schaeffer, 2002). Les intentionnalités des réalisateurs ne sont pourtant pas toujours tranchées : ainsi, Cary Fukunaga explique dans un entretien réalisé en 2009 au sujet de Sin Nombre, film de fiction, qu'il avait pour intention de «documenter» la frontière sud du Mexique, très peu médiatisée comparée à la frontière nord. Pour autant, Sin Nombre et No Country For Old Men relèvent de régimes fictionnels, qui matérialisent des imaginaires dont il s'agit d'interroger la portée cognitive.

-La portée cognitive de la fiction cinématographique

Si toute opération de description référentielle, cinématographique ou géographique, relève d'une construction de l'imagination, d'une élaboration mentale, les films de fiction, pour lesquels « la question de la référentialité ne compte pas » (Schaeffer, 2002), peuvent-ils contribuer à la production de connaissances géographiques ?

La proposition de Christine Chivallon selon laquelle «l'imaginaire est à l'œuvre dans toute élaboration des formes sociales » (Chivallon, 2008, p. 80) autorise à considérer la portée cognitive de l'imagination, mise en œuvre dans la fiction. Jean-Marie Schaeffer explique que «si, dans certains processus cognitifs, des représentations imaginaires jouent un rôle indispensable, alors la question de la portée cognitive d'une représentation ne saurait être réduite à celle de sa force référentielle » (Schaeffer, 2002). Le cinéma de fiction, non-réferentiel et non-vérifonctionnel, pourrait ainsi être intégré à un projet cognitif en géographie. Cette démarche est appliquée par Jean-François Staszak, qui, dans une étude des films de fiction hollywoodiens mettant en scène des actrices d'origine chinoise, explique que «si les films nous intéressent, c'est non seulement parce qu'ils donnent accès aux représentations de ceux qui les ont produits, mais aussi parce qu'ils influencent ou ont influencé les représentations et les comportements - notamment géographiques - du public qui les a vus, et donc qu'ils constituent des facteurs des évolutions sociales et spatiales»(Staszak, 2011). Le terme de représentation renvoie ici aux expressions d'une culture, qui médiatise, à une époque donnée, les rapports sociaux et les relations des 
sociétés à l'espace. «Donner accès » à ces expressions culturelles est une des fonctions cognitives possibles de la fiction, sans que la fiction ne se réduise à un usage cognitif ${ }^{9}$.

En projetant des mondes désirables ou indésirables, notamment des utopies, la fiction permet également de «dynamiser notre univers mental » (Schaeffer, 2002). Ainsi, la fiction, en tant que « modèle virtuel » (ibid.), ouvre des hypothèses, dont il ne s'agit pas de vérifier la validité, la justesse ou la fausseté, mais dont l'expérimentation est porteuse de sens. Dans le cas des films étudiés, le spectateur expérimente des modèles virtuels, des situations simulées, de fuites et de traversées frontalières, sur le mode de l'immersion. L'expérimentation du spectateur peut «intervenir sous de multiples formes dans [ses] interactions futures avec la réalité, puisqu'elles mettent à [sa] disposition des boucles de traitement mental endogène qu'[il] $\mathrm{p}$ [eut] réactiver à volonté chaque fois qu'[il se] trouv[e] face à un domaine analogique pertinent. [...] C'est donc paradoxalement en nous ouvrant l'espace des possibles, que la fiction nous permet de mieux maîtriser le réel »(ibid.). Faire l'expérience de ces univers fictionnels aurait ainsi une portée cognitive : «les modèles fictionnels sont en effet susceptibles [...] de nous amener à suspendre notre jugement, à peser des évaluations axiologiques, à simuler des réactions affectives [...] sans que ces expérimentations ne soient directement sanctionnées par le réel. [...] Mais ils peuvent aussi nous permettre d'opposer un contremodèle, un monde alternatif, à la réalité instituée, comme c'est le cas dans les fictions utopiques » (ibid.).

A partir de ces analyses, notre propos est ici d'étudier la pertinence de l'application analogique des modèles fictionnels développés dans Sin Nombre et No Country For Old Men pour construire et «dynamiser » les modèles géographiques et cartographiques appliquées aux frontières et aux déplacements frontaliers.

-Faire l'expérience des étendues frontalières : le Mexique, état-frontière dans

9 Jean-Marie Schaeffer explique à ce propos: «si je soutiens l'hypothèse d'une potentialité cognitive de l'imaginaire ou de la fiction je ne plaide pas pour autant en faveur d'une théorie du «tout cognitif». Je crois au contraire que la réduction des représentations à la fonction cognitive n'est nullement justifiée » (Schaeffer, 2002). 


\section{Sin Nombre}

Sin Nombre et No Country For Old Men sont deux films de fiction qui ont pour point commun de montrer des espaces frontaliers depuis le point de vue de personnages en fuite.

Cary Fukunaga dans Sin Nombre, tente ainsi de décrire et de figurer, selon un registre à la fois référentiel et non-référentiel, un vaste espace frontalier allant du Honduras au Nord du Mexique. Les scènes, fictives, sont filmées in situ et jouées par des acteurs professionnels et nonprofessionnels. Tout l'espace parcouru par Sayra, son père et son oncle est un espace frontalier, semé de dangers, de barrières, et de temps d'attente plus ou moins longs. Le Mexique est décrit comme un véritable étatfrontière. Sayra et sa famille restent longuement bloquée à la gare frontalière de Tapachula, dans l'état du Chiapas, en attente d'un train qui les mènera à Mexico. L'espace du tracé frontalier entre les États-Unis et le Mexique, topos du cinéma contemporain américain (Mains, 2004), perd de sa centralité dans Sin Nombre, qui montre toute l'épaisseur de la frontière sud-mexicaine et du Mexique comme état-frontière pour les migrants issus d'Amérique centrale et latine. Alejandra Carrillo rapporte les statistiques officielles de l'Etat mexicain sur le passage de la frontière sud, et plus précisément le nombre de migrants illégaux qui y sont détenus chaque année : « dans les onze points d'internement officiels, distribués tout au long de cette ligne de 1149 kilomètres séparant le Mexique du Guatemala et du Belize, sont enregistrées en moyenne 2 millions d'entrées par an. » (Carillo, 2008). En traversant le Mexique sur le toit des trains reliant Tapachula à Mexico, les migrants sans autorisation de séjour au Mexique sont en effet souvent arrêtés, placés en rétention et expulsés, mais aussi rackettés par la police mexicaine et des groupes criminels. Alejandra Carrillo précise que le contrôle des migrants au Mexique s'étend tout au long de l'axe reliant Tapachula à Mexico « où se répartissent 25 postes de contrôle militaire et 21 postes de contrôle migratoire [...]. Il y a donc un éclatement spatio-temporel des contrôles aux frontières puisque ceux-ci s'étalent dans toute la région sud du pays et pas seulement à la ligne frontalière » (ibid.). Ces aménagements disciplinaires relèvent d'une politique d'externalisation de la frontière entre les États-Unis et le Mexique, 
mise en place principalement à partir de 2001. Déplaçant la ligne d'opposition classique entre Nord et Sud et au-delà la notion même de frontière, Sin Nombre montre une vaste étendue frontalière, située entre deux tracés linéaires des frontières mexicaines. La figure d'une étendue située dans un entre-deux est également mise en scène dans No Country For Old Men.

\section{-L'entre-deux frontalier dans No Country For Old Men}

Au sujet de Cormac McCarthy, auteur de No Country For Old Men à partir duquel le film éponyme a été réalisé, Florence Césari-Stricker décrit un « romancier des déclins, des confins et des crépuscules. Cormac McCarthy situe toujours ses fictions dans un intervalle : [...] limite entre deux frontières » (Cesari-Stricker, 2008, p. 6-7). L'étendue frontalière, qui se déploie entre deux (ou plusieurs) « portes » d'entrée et de sortie, serait ainsi un seuil, c'est-à-dire un espace de transition où l'on n'est pas encore « dedans », ni plus tout à fait « dehors ».

Dans No Country For Old Men, Llewelynn tente de fuir à travers une étendue frontalière comprise entre la porte des États-Unis ouvrant sur le Mexique et la porte du Mexique ouvrant sur les États-Unis. Lorsque Llewelynn arrive à la «frontière », celle-ci est délimitée par un poste douanier où est mentionné « United States Border », qui s'ouvre ensuite sur un pont, construit au-dessus du Rio Grande. Une succession de plans montre Llewelynn sur le pont, avec en arrière-plan les postes-frontières tantôt étatsunien, tantôt mexicain, suivant l'emplacement de la caméra. La frontière se dédouble en deux passages, qui se prolongent par un seuil. Elle est aussi mise en perspective par la profondeur de l'espace sous le pont, qui s'étend jusqu'au fleuve. Llewelynn trouve d'ailleurs dans cette profondeur un refuge provisoire, en jetant la mallette pleine d'argent qu'il transporte par-dessus le pont, pour la cacher et ainsi se protéger de ses agresseurs.

Les choix fictionnels de Sin Nombre et No Country For Old Men permettent de faire l'expérience de frontières en tant que vastes étendues spatiales, déployées dans des entre-deux. Les espaces frontaliers sont perçus depuis la perspective de leur franchissement. Cette perspective est renforcée par une série de dispositifs cinématographiques, qui empruntent aux 
dispositifs de la cartographie et contribuent aussi à les interroger.

2- Visualiser et figurer les frontières mobiles

Teresa Castro rappelle combien le cinéma est « un art générateur d'espaces »(Castro, 2011, p. 8); il permet de décrire, de constituer des espaces, selon un régime de visibilité diagrammatique partagé avec la carte. Sébastien Caquard et Fraser Taylor (2009) montrent par ailleurs combien la cartographie animée emprunte à l'image cinématographique, toujours soumise au temps.

Le cinéma crée en effet un espace-temps animé, selon trois principes fondamentaux. L'image cinématographique est d'abord «dans le temps » (Dufour, 2009, p. 34-35), c'est une « image qui dure et donc se transforme » (ibid., p. 31); la durée de l'image est délimitée par un plan. Ensuite, l'image est un espace, délimité par un cadre, qui peut être mobile. Enfin, un film est une succession d'images, c'est-à-dire de plans et de cadres. Le montage permet de mettre en relation les images, pour créer un espace-temps singulier, où se multiplient les points de vue, les échelles, les durées. La frontière est donc saisie dans le temps et depuis les mouvements des personnages. Ces éléments narratifs sont matérialisés par plusieurs procédés filmiques.

\section{-La frontière en perspective}

Les vues d'ensemble, saisies par des plans fixes en plongée, avec un point de fuite central, sont très nombreuses dans les deux films étudiés, et plus généralement dans les films classés sous l'appellation des road movies. Dans No Country For Old Men, nombreux sont les plans de routes traversant le désert, orientées vers un point de vue central (cf. figure 1). Dans Sin Nombre, la caméra placée sur le toit du train en mouvement, dans lequel voyagent Sayra et sa famille, filme les personnages de face ou de dos; la composition de ces plans converge toujours vers un point de fuite situé dans le fond. A Tapachulpa, dans la gare de marchandises, de nombreux plans fixes reproduisent le même procédé (cf. figure 2). 


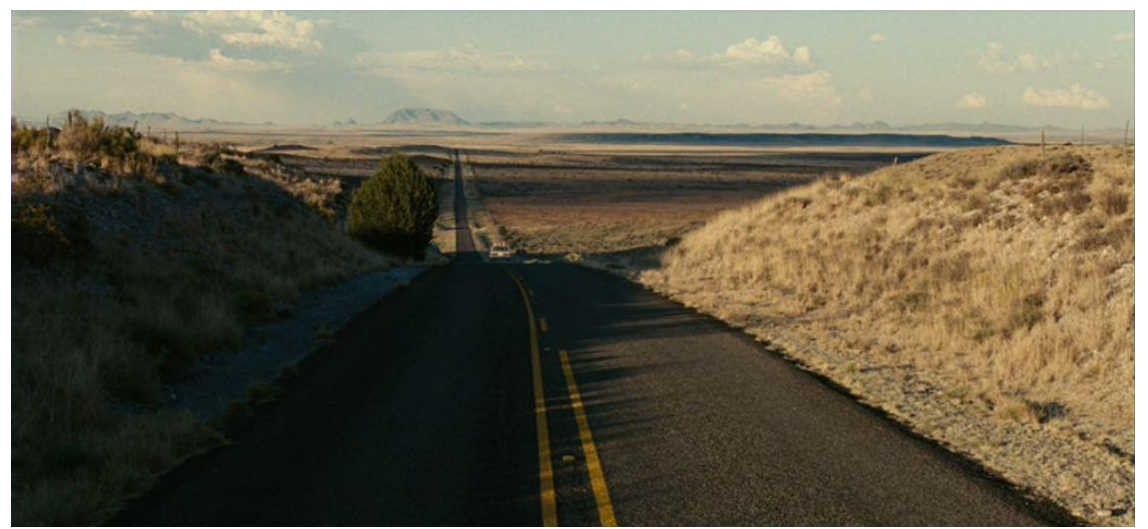

Figure 1- Vue d'ensemble et point de fuite, arrêt sur image, No Country For

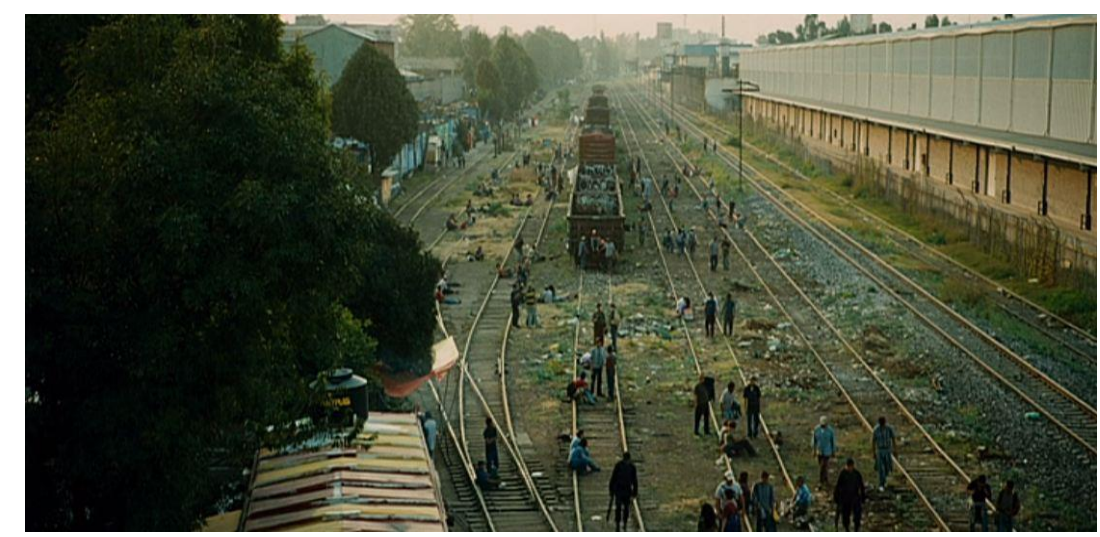

Old Men

Figure 2- Vue d'ensemble plongeante et point de fuite, arrêt sur image, Sin Nombre

La plongée reproduit une position de surplomb qui place le spectateur dans une position comparable à celle du lecteur d'une carte. Si les cartes sont construites à partir d'un «paradigme zénithal » (Castro, 2011, p. 40), soit d'un angle de vue vertical, le point de fuite dépend d'un angle de vue oblique. Mais la vue oblique partage des caractéristiques communes avec la vue verticale, notamment car elle permet de dominer l'espace du regard. A la différence de la vue verticale, elle ouvre une perspective, particulièrement signifiante pour comprendre les fonctionnements frontaliers.

Dans une analyse filmique de Paris qui dort réalisé en 1923 par René Clair, Tom Conley montre l'importance de la vue d'ensemble structurée par un point de fuite ( vanishing point ») dans la construction de plans qui fonctionnent comme des cartes («map-like shots ») (Conley, 2007, p. 37). A partir du concept d' «objet de perspective» emprunté au 
psychanalyste Guy Rosolato, Tom Conley montre que la Statue de la Liberté dans la vue en surplomb de la Seine dans Paris qui dort est « un objet de perspective », c'est-à-dire « un signe de représentation de l'inconnu » (ibid., p. 221), une cause et un objet du désir. Les frontières, plutôt que des lignes tracées entre deux mondes connus, seraient des espaces d'entre-deux, entre monde connu et monde inconnu, matérialisé par le point de fuite ou par des « objets de perspective ».

L'expérience d'un manque, contenu dans le désir de l'inconnu, est mise sous tension dans l'image, comme nous venons de le voir, et hors de l'image, dans le hors-champ.

-Les images cinématographiques de la frontière ouvertes sur un ailleurs

Le cadre de l'image cinématographique n'est pas une clôture, qui enferme le visible. Il y a «autre chose », un prolongement au-delà de l'image visible, qui peut, par un mouvement de caméra, entrer dans le champ. Le champ est ainsi toujours prolongé par le hors-champ, qui n'est pas visible mais pourtant présent. «L'opération de cadrage consiste [...] à engendrer physiquement un espace. Mais, au cinéma, à la différence de la peinture, comme les images sont en mouvement, ce qui n'est pas présent dans le cadre n'est pas pour autant absent. Le hors-champ désigne ce qui n'est pas cadré (ce qu'on entend pas et ne voit pas), et qui pourtant « insiste » ou « subsiste » dans le présent » (Montebello, 2008, p. 30).

La perspective d'une étendue frontalière, qui se prolonge par un autre monde, motive les migrations. Le hors-champ est une dimension spatio-temporelle fondamentale dans la géographie des migrations et dans le fonctionnement des frontières. Dans la gare de Tapachula, le père de Sayra déplie et commente une carte de l'Amérique du Nord, où figure le tracé du chemin de fer qu'ils souhaitent emprunter vers les Etats-Unis. Sayra demande à son père de localiser le New Jersey, leur destination finale. Un gros plan montre alors le haut de la carte où est représenté en blanc le sud des États-Unis, on entend la voix du père qui précise «c'est plus haut », en faisant un geste de la main dont l'amplitude est coupée par le cadrage rapproché de la caméra. 


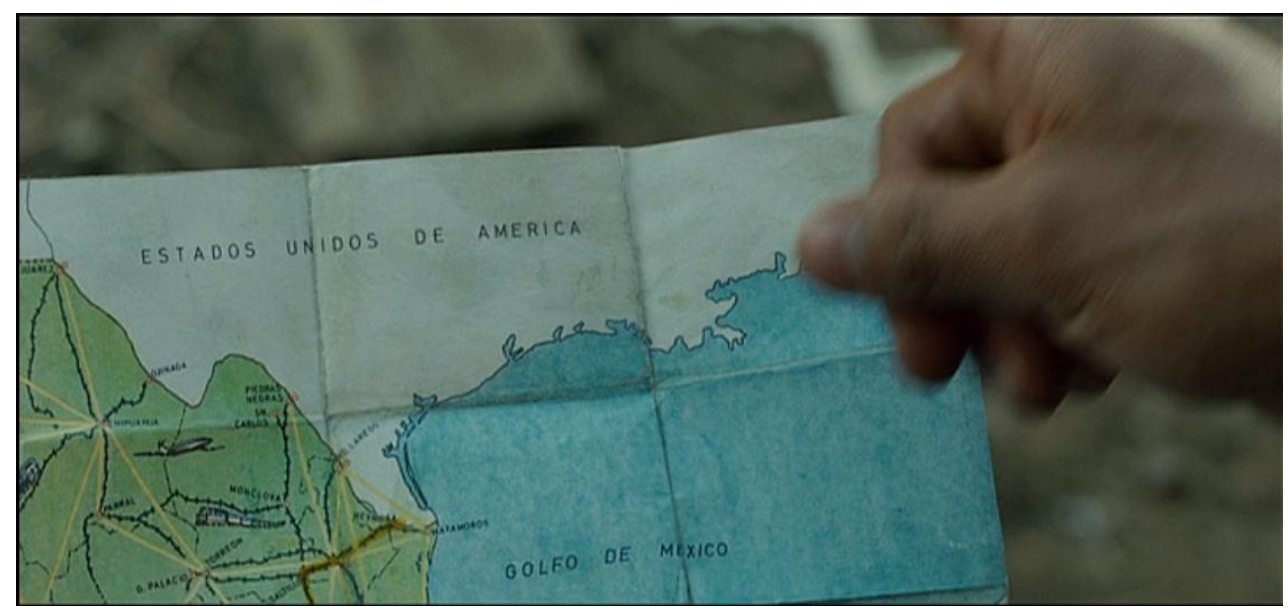

Figure 3- Carte topographique et hors-champ, arrêt sur image, Sin Nombre

Terra incognita, les États-Unis sont représentés en blanc. Le New Jersey ne figure pas sur la carte et le mouvement de la main du père est coupée par le cadre (cf. figure 3). La destination finale est en hors-champ. Le hors-champ est à la fois un au-delà invisible et incertain de la frontière, où aucune trajectoire n'est dessinée, et un lieu omniprésent vers lequel tendent tous les mouvements.

-Introduire le hors-champ dans la cartographie des itinéraires migratoires transfrontaliers

Les cartes de trajectoires migratoires montrent souvent, selon des rythmes différenciés pour les cartes animées (Poli, 2007), des tracés entre point de départ, points de transit et point d'arrivée, séparés par des tracés frontaliers. Ces cartes insistent plus sur les nœuds que sur l'espace-temps du voyage, situés entre ces nœuds. Dans le cas des franchissements frontaliers terrestres ou maritimes ${ }^{10}$, l'espace-temps du voyage est fait d'expériences singulières, souvent très significatives pour les individus (Green, 1999). La littérature sur les migrations africaines vers l'Europe montre combien le voyage, à travers des espaces-temps frontaliers profonds et périlleux, est central dans les expériences migratoires des individus (Arab, 2007; Bennafla, Peraldi, 2008; Pian, 2009; Pliez, 2010); les œuvres cinématographiques, documentaires et fictionnelles, sur les expériences des

10 Les déplacements en avion posent la question du franchissement des frontières différemment ; les frontières sont alors nodales, matérialisées par les aéroports. 
traversées sont également nombreuses ${ }^{11}$. En considérant la frontière comme un espace à parcourir, entre deux espaces hors-champ, on peut imaginer figurer l'espace topographique à franchir, en le situant entre deux espaces présents mais situés dans l'ombre. Dans une perspective dynamique, il s'agirait de réduire progressivement l'épaisseur de l'espace frontalier, en plongeant dans l'ombre les espaces déjà parcourus, ou, à l'inverse, en augmentant l'épaisseur de la frontière, en éclairant les espaces encore à parcourir. Selon un procédé comparable, les premières images de No Country For Old Men montrent de vastes étendues désertiques frontalières au crépuscule, dont la profondeur de champ augmente au fur et à mesure que le soleil se lève. Cette proposition de cartographier l'entre-deux frontalier, au rythme de la traversée des individus, se situe dans la perspective des « cartes-itinéraires », qui «fourni[ssen]t une représentation du territoire dans laquelle celui-ci n'est pas considéré indépendamment des pratiques qui s'y déploient [...] mais au contraire défini dans sa structure même par les engagements pratiques de ceux qui y inscrivent leurs déambulations »(Besse, 2010, p. 7).

Ainsi, l'étude des images cinématographiques amène à s'interroger sur les modes de visualisation de l'entre-deux frontalier. La multiplication des points de vue et des cadrages, constitutive du cinéma, ouvre des questionnements sur les caractéristiques multidimensionnelles de la frontière.

-Multiplier les points de vue pour analyser la frontière mobile

Les premiers plans de No Country For Old Men, évoqués précédemment, sont filmés depuis une multiplication de points de vue. La ligne d'horizon, plus ou moins haute dans l'image, est parfois parallèle au tracé d'une clôture en barbelé, parfois coupée par la clôture. Celle-ci court entre des espaces sans fin, annonçant une frontière linéaire qui peine à

11 Des festivals de films sur les migrations, et plus particulièrement sur les voyages migratoires, se développent dans de nombreux pays, on peut citer par exemple le festival LampedusaInFestival sur les migrations, qui se tient à Lampedusa ou encore le festival culturel Migrant'Scène organisé par la Cimade en France. Le film récent de Moussa Touré La Pirogue (2012) reprend le thème des traversées de fortune de la Méditerranée par des migrants et fait écho à de nombreuses œuvres, fictionnelles et documentaires qui traitent spécifiquement des expériences du voyage, par exemple le film Harragas, réalisé par Merzak Allouache, sorti en 2010 en France. 
délimiter l'étendue.
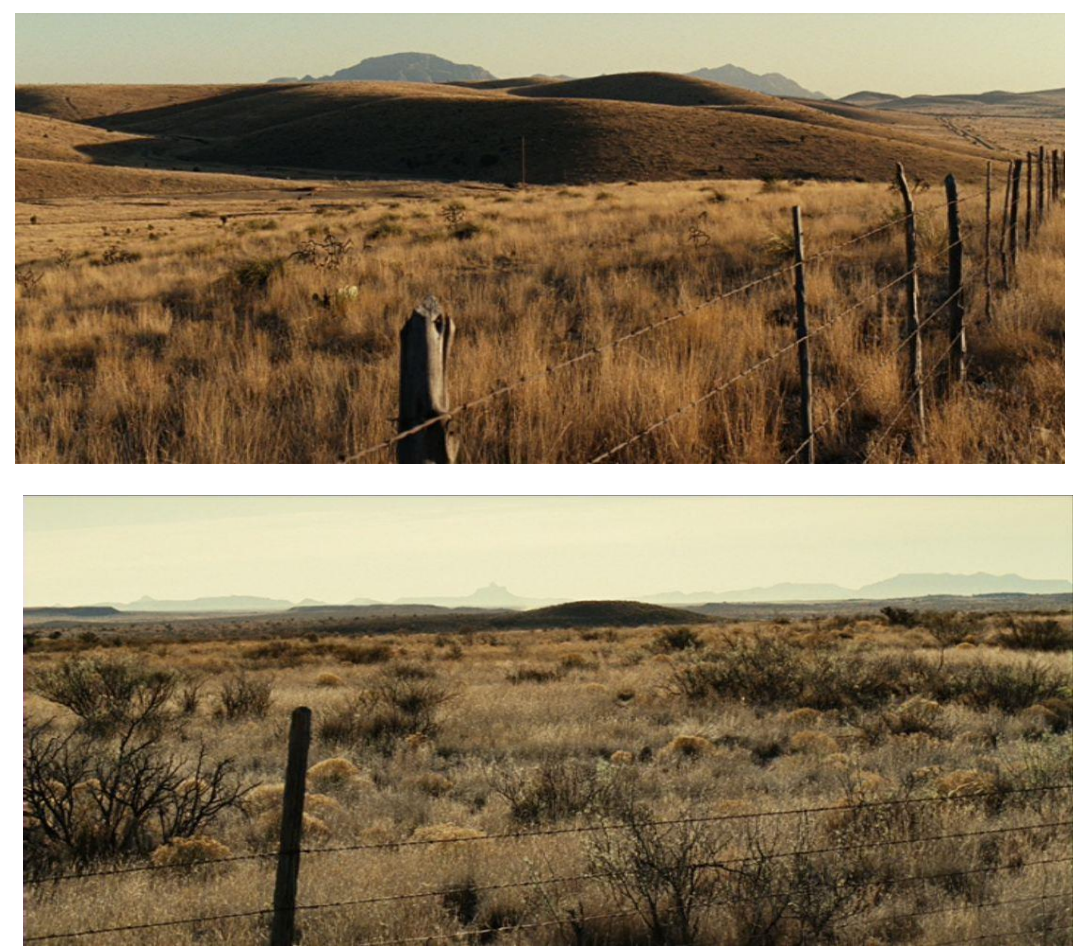

Figure 4- Succession de plans d'ensemble de l'étendue frontalière, arrêts sur image, No Country For Old Men

Si les plans d'ensemble servent une visée descriptive, leur succession, selon des points de vue distincts, relève du diagramme. Pris séparément, les premiers plans de No Country For Old Men, peuvent décrire soit l'ouverture, soit la fermeture des étendues frontalières. Or, il serait réducteur d'opposer un type de plan à un autre ; c'est bien dans la mise en relation de ces plans, par le montage, que l'effet de réel et la complexité de l'objet géographique frontalier apparaît. Les plans, les points de vue et les échelles changent ; ils donnent à voir une étendue multidimensionnelle, dont l'ouverture et le cloisonnement coexistent et évoluent.

La séquence inaugurale de Sin Nombre est éloquente pour saisir l'importance du montage dans la construction d'un objet spatial multidimensionnel. Le premier plan du film montre le paysage d'un sousbois en automne, où un chemin court vers un point de fuite. Ce paysage est filmé dans un plan d'ensemble avec un travelling avant. Ce procédé 
accentue l'impression de participer à la scène et d'avancer sur le chemin. Suit un plan d'ensemble avec un travelling avant de Casper, membre de la Mara Salvatrucha, assis immobile dans une pièce confinée et délabrée. La caméra est placée ensuite dans le dos de Casper. Un plan en plongée montre la tête de Casper vue de dos ; dans l'arrière-plan, apparaît le paysage du sous-bois. Le spectateur comprend alors que le paysage est une photographie murale. L'illusion de profondeur de la première image tombe à plat! Le dernier plan de la séquence montre Casper de dos qui se lève, se dirige vers le mur-photographie, bifurque vers la gauche et sort de la pièce, en même temps qu'il sort du champ.

Cette séquence rappelle les multiples dimensions de la frontière : en fonction du point de vue adopté, elle peut appeler un ailleurs et un déplacement, mais elle peut aussi être un mur, faisant disparaître toute illusion de la franchir. C'est aussi la versatilité du voyage qui est abordée ; celui-ci peut brutalement s'arrêter face à l'irruption d'un mur et à tous les périls qui peuvent survenir sur le chemin de la migration ${ }^{12}$. Ainsi, l'étendue et la profondeur frontalière ne peuvent être perçues qu'au prix de l'abandon d'un cadrage fixe et unique, autrement dit, au prix d'un décentrement du regard.

-Cartographier les déplacements frontaliers

Plutôt que de se situer de tel ou tel «côté » de la frontière pour interroger son fonctionnement, on peut penser à multiplier les points de vue, en se déplaçant sur l'étendue même de la frontière. La méthodologie ethnographique multi-située a été notamment mise en œuvre par Olivier Pliez pour analyser les géographies migratoires au Sahara (Pliez, 2011). Cette méthodologie semble particulièrement adaptée pour construire une géographie des frontières depuis le point de vue des individus en mouvement, recueillis à différents moments et lieux de leur voyage. L'épaisseur de la frontière n'est pas seulement mesurable en terme de

12 Les deux films mettent en scène des trajectoires croisées, qui soit, s'arrêtent brutalement en raison de morts violentes, soit, poursuivent leur cours. Casper et Llewelynn meurent tous deux à la limite de la possibilité d'une nouvelle vie : sur la rive mexicaine du Rio Grande pour l'un, au seuil des États-Unis, à El Paso pour l'autre. Sayra est la seule qui atteint les États-Unis, son père meurt au Mexique abattu par la police mexicaine sur le toit d'un train, tandis que son oncle est expulsé du Mexique vers le Honduras. 
distance entre les points de départ, de transit et d'arrivée; elle peut également se mesurer depuis l'expérience et la perception des individus, qui tentent de la traverser. Ces aspects méthodologiques rejoignent les dispositifs mobilisés à l'échelle intra-urbaine et depuis un point de vue artistique par Mathias Poisson (2010) pour produire des «graphies du déplacement ». Mathias Poisson, «artiste promeneur » dessine des cartes, en traversant des villes à pied, qui «témoigne[nt] de sensations, de préoccupations et d'observations survenues lors de ces déambulations » (Poisson, 2010, p. 105). Les oeuvres de l'auteur tentent de répondre à des questionnements, également au cœur des réflexions sur la "graphie» des déplacements frontaliers : «comment un espace perçu en mouvement peutil être dessiné ? Comment un trajet est-il mémorisé ? (ibid.). La prise en compte des expériences individuelles et collectives, "chemin faisant», participe d'une définition de la frontière en tant qu'objet géographique instable et en devenir.

3-La frontière produite « chemin faisant ».

Les déplacements et les parcours migratoires contribuent, au fur et à mesure de leur progression, à faire évoluer l'étendue frontalière, qui se constitue «chemin faisant». Plusieurs procédés filmiques viennent renforcer cette construction progressive et éphémère. Les mentions évanescentes de toponymes ou encore l'apparition à l'écran de cartes topographiques illisibles, traduisent l'instabilité fondamentale de l'étendue frontalière.

- Des repères topographiques évanescents

Le parcours des protagonistes dans Sin Nombre est ponctué par une série de noms de lieux ${ }^{13}$, surimposés aux images, rappelant les toponymes qui figurent sur une carte. Ces noms sont ajoutés au montage et produits depuis un off ${ }^{14}$. Écrire des noms de lieux sur l'image est un procédé de

\footnotetext{
13 « Guatemala ; Mexico Frontera » (17'), «Tierra Blanca, Veracruz » (49'), « Reynosa, Tamaulipas » (1'15).

14 Le « off » désigne un lieu et/ou un temps, différents de ceux de la diégèse, d'où provient
} 
localisation peu cinématographique, au sens où l'image, et non le texte, est première au cinéma. Néanmoins, la mention des lieux n'est pas statique, elle suit le mouvement des images et disparaît avec le plan suivant. Quand Sayra et sa famille traversent un fleuve en pirogue entre le Guatemala et le Mexique, un travelling en plongée suit leur avancée. Quand la caméra en mouvement filme la rive guatémaltèque, le toponyme «Guatemala » apparaît en haut à gauche de l'écran, puis disparaît. Le travelling se poursuit vers l'autre rive et la mention «Mexico Frontera » apparaît à son tour, pour ensuite disparaître. Plusieurs toponymes dans Sin Nombre se surimposent à une image en mouvement (traversée du fleuve, mouvement du train) ; quand le texte est surimprimé sur un plan fixe, le plan est cadré en plongée, avec une importante profondeur de champ. Bien plus que des points fixes, ces noms de lieux désignent des étendues à parcourir, et par conséquent des espaces voués à défiler, à se dissoudre et à disparaître au fur et à mesure du voyage.

L'irruption de toponymes dans les décors du film, sur les costumes des personnages en mouvement, puis leur disparition, relève d'un procédé comparable: les cartographies filmiques construites sont animées et évanescentes. Les noms de lieux prononcés dans les dialogues participent, par ailleurs, à l'élaboration de cartes audibles, éphémères et invisibles. La bande-son filmique hors-dialogue (musique, bruits) comporte également des éléments symboliques de repérage topographique : le souffle du vent pour le désert dans No Country For Old Men, le bruit du train pour le voyage dans Sin Nombre. La bande-son n'est pas forcément « in », c'est-à-dire que sa source ne correspond pas forcément à ce qui apparaît dans le champ, on parle alors d'un son « off » (dont la source se trouve dans un lieu et/ou un temps extra-diégétique) ou d'un son «hors-champ» (dont la source est située dans la continuité non-visible du champ).

Rendre les repères topographiques évanescents est un dispositif qui contribue à signifier l'instabilité du voyage en train de se faire. Les apparitions à l'écran de cartes topographiques participent de la même fonction.

un son (la voix off par exemple) ou ici un texte. 
-Ouvrir des chemins

Dans Sin Nombre et No Country For Old Men, le traitement filmique des cartes topographiques, tant par le cadrage des plans que par les éléments narratifs, souligne leur relative inutilité à maîtriser et à fixer une fois pour toutes l'étendue frontalière. Jean-Marc Besse rappelle à ce sujet que la carte, bien qu'étant une synthèse graphique stabilisée à un moment donné, est relative et instable; elle est toujours amenée à être modifiée. « La rectification est selon Christian Jacob, le mouvement fondamental de la cartographie. [...] En tant que produit de savoir, la carte est toujours un moment dans un mouvement de figuration du territoire dont elle n'offre à chaque fois qu'une version. Elle est comme une coupe instantanée dans la dynamique temporelle d'un projet de connaissance orienté vers le territoire. »(Besse, 2008). En plus de sa relativité historique, la carte est aussi amenée à être modifiée en fonction des changements de l'objet qu'elle tente de figurer. Dans le cas de la cartographie des déplacements frontaliers, leur caractère périlleux ${ }^{15}$ et leurs changements constants impliquent d'imaginer et de construire des séries de cartes animées, en évolution.

Dans les films étudiés, les cartes statiques qui apparaissent à l'écran contrastent avec le vécu des personnages en mouvement. Quand la trajectoire de Sayra commence à s'écarter de la route tracée à l'avance par son père pour se rendre aux États-Unis, ce dernier remontre à sa fille la carte topographique qu'il lui avait montrée au début du voyage, à Tapachula. Il distingue l'espace déjà parcouru de l'espace encore à parcourir. La carte a ici un rôle d'injonction, elle montre la route à suivre, tracée par l'autorité paternelle. Cette carte se révèle, dans la suite du film, inutile, en contradiction avec le cours des événements qui affectent Sayra. Ses bifurcations, forcées ou choisies, ne figurent pas sur la carte, tracée a priori. Les repères topographiques ne dispensent ni de se perdre ni de se sentir perdu, dépassé par le cours du temps et l'urgence des événements. Tom Conley explique ainsi que la carte à l'écran peut être le «point de départ

15 Florence Césari-Stricker explique que le péril est défini à la fois comme une épreuve (periculum en latin) et comme l'expérience d'une traversée (per, la racine latine de periculum, signifie aller de l'avant, traverser), (Cesari-Stricker, 2008, p. 6-7). 
d'un itinéraire interprétatif » (" a point of departure for an interpretative itinerary »), (Conley, 2007, p. 208) ; loin de fixer un cadre stable, la carte ouvre des possibles. Du point de vue de la narration dans Sin Nombre, la carte topographique est effectivement un point de départ à partir duquel les parcours des personnages divaguent et bifurquent. Dans No Country For Old Men, le bureau du shérif Bell est recouvert de cartes topographiques à différentes échelles. Lorsque le shérif s'apprête à quitter son bureau pour partir enquêter sur le terrain, il est filmé en plan moyen sur fond d'une carte topographique quadrillée du Texas (cf. figure 5). La carte, objet de savoir et de pouvoir, est associée à l'autorité, qui dans le cas du shérif Bell, est limitée. Le shérif arrive toujours sur les lieux des crimes après-coup, quand il ne refuse pas carrément de se déplacer, blasé par un contexte criminel qu'il accepte, tant bien que mal, lui échapper.

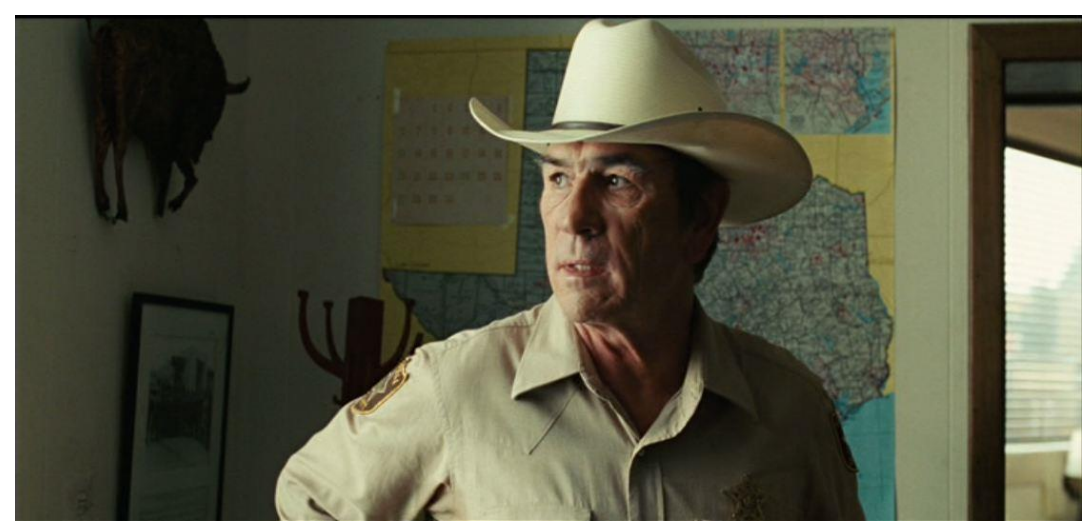

Figure 5- Le shérif Bell et la carte du Texas, arrêt sur image, No Country For Old Men

Par ailleurs, la carte topographique que Llewelynn regarde avant de s'élancer dans le désert, représente l'espace frontalier local où se situe l'action, avec une figuration du tracé de la frontière en pointillé. La manière dont la carte est filmée, dans la pénombre, éclairée par un petit faisceau lumineux d'une lampe de poche située dans le hors-champ, ne laisse distinguer aucun toponyme (cf. figure 6). Le plan ne dure que quelques secondes. L'éclairage par intermittence annonce la fragilité des repères pour se déplacer dans cette immense étendue frontalière désertique. John Bruns, dans un article sur la place de la carte dans No Country For Old Men, 
montre combien «le terrain topographique [de la frontière] est instable » (an « unstable topographic field») et hostile (Bruns, 2011), en insistant sur la menace qui pèse sur Llewelynn, toujours rattrapé par Anton Chigurh. L'auteur explique que la maîtrise de l'espace est du côté du personnage Anton Chigurh, et non de Lelwelynn ou encore du shérif Bell qui pourtant sont entourés de cartes. Anton Chigurh dispose uniquement d'un appareil qui bipe de plus en plus vite, à mesure qu'il s'approche de l'émetteur dissimulé dans la valise de billets que transporte Llewelynn. Ses apparitions et disparitions soudaines, toujours au bon endroit, déstabilisent les repères attendus du spectateur. Il semblerait qu'Anton Chigurh, qui traque Llewelynn, se déplace uniquement au bruit de l'appareil, sans repères cartographiques. Les images cinématographiques fictionnelles permettent ici de « se soumettre à la capacité du cinéma de dénaturaliser notre perception de l'espace » («to submit oneself to the potential of cinema to estrange you from your sense of location ») (Bruns, 2011).

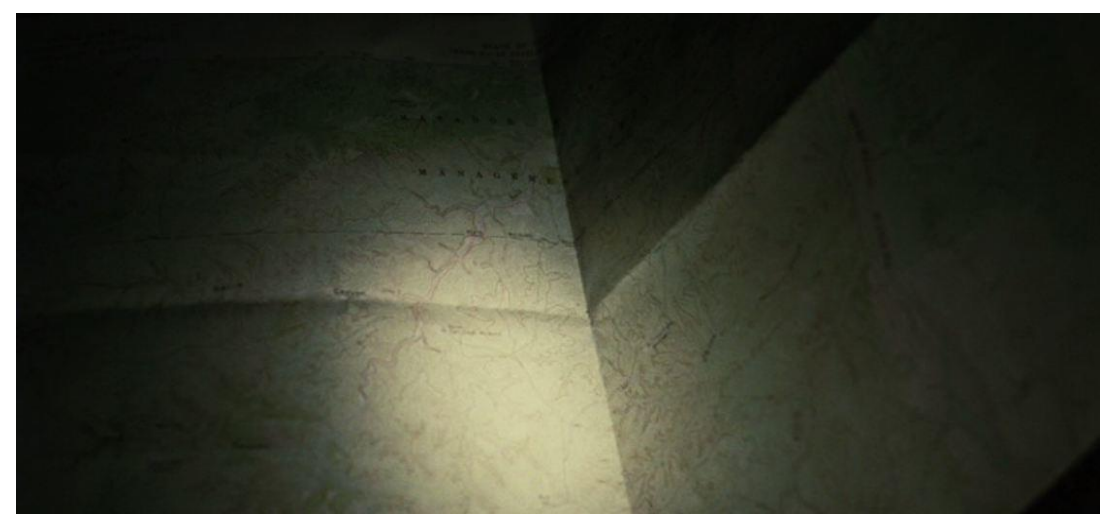

Figure 6- La carte illisible, arrêt sur image, No Country For Old Men

Les cartes topographiques, qui permettraient de tracer des routes, soustraites au temps et à la contingence des événements, se trouvent confrontées aux pratiques spatiales et aux histoires mouvementées des personnages. Gilles Tiberghien, dans un article sur l'hodologie ${ }^{16}$, distingue la route du chemin : «la route et les termes qui lui sont apparentés sont du côté de la construction et de la surface parcourue; le chemin du côté du

16 L'hodologie est définie par John Brinckeroff Jackson comme « la science ou l'étude des routes » (2003, p. 79). Gilles Tiberghien complète cet élément de définition «L'hodologie s'intéresse au route, aux rues, aux différentes voies de communication : cela signifie aussi qu'elle tient compte de ceux qui s'en servent, qui les « empruntent» le temps d'un trajet plus ou moins long. Mais « route» n'est peut être pas le mot plus adéquat. On peut lui préférer « chemin ».» (Tiberghien, 2004, p. 8). 
mouvement et du processus » (Tiberghien, 2004, p. 9). Cette réflexion est construite à partir des écrits anglophones de John Brinckeroff Jackson (2003), qui utilise le terme «way», traduit par «chemin» en français : «way signifie non seulement chemin, mais encore [...] projet et façon » (Brinckeroff Jackson, 2003, p. 79). Le père de Sayra avait calqué le chemin qu'il souhaitait prendre à une route prédéfinie, qui correspondait au tracé de la voie de chemin de fer. Or, le cours des événements implique des évolutions de trajectoires, que nulle carte ne pouvait saisir a priori.

Étendue parcourue du regard et traversée par des corps en mouvement, la frontière est un espace d'entre-deux qui crée des devenirs, par définition imprévisibles, allant de la mort pour certains, à la création d'une vie nouvelle, inédite pour d'autres.

\section{Conclusion}

Au sujet de l'espace saharo-sahélien, Armelle Choplin et Olivier Pliez expliquent la difficulté de cartographier les déplacements qui s'y déploient, ainsi que «toutes les aspérités -spatiales et temporelles d'ordre politique, policier, pécuniaire...- qui jalonnent les itinéraires empruntés par les migrants » (Choplin, Pliez, 2011). Le Sahara ou encore les confins nordaméricains sont des espaces frontaliers particulièrement complexes à figurer, tant leurs fonctions de barrière ou d'interface se déplacent, évoluent en fonction des flux et des trajectoires migratoires, eux-mêmes évolutifs. Les recherches en géomatique sur les cartes animées et sur la figuration des processus de diffusion et de changements spatio-temporels, ont largement contribué à introduire une définition dynamique de l'espace. En plus de la difficulté de représenter des données spatio-temporelles quantitatives complexes, s'ajoute l'ambition de construire des cartes qualitatives où puisse figurer l'expérience des déplacements et des traversées frontalières. Les migrations transfrontalières sont appréhendables en tant que flux, trajectoires mais aussi en tant qu'expériences individuelles et collectives. Les « cartes sensibles » (Olmedo, 2011), les « graphies du déplacement » (Poisson, 2010) entre sciences et arts, ouvrent des perspectives méthodologiques et analytiques particulièrement fructueuses pour la 
géographie des frontières.

En plus de la visée cognitive, étudier les frontières depuis les pratiques et les expériences de mobilité qu'elles génèrent, a aussi une portée politique. Une pensée qui ne ferait qu'opposer la frontière et les mouvements migratoires, selon un strict dualisme, relèverait d'un point de vue politique centralisé, de politiques étatiques disciplinaires, ayant pour objectif le contrôle des mouvements migratoires. Conçue comme l'enveloppe protectrice du corps de la nation, la frontière, pièce essentielle des idéologies nationalistes, est naturalisée et figée. Le décentrement et la critique de ce point de vue autorisent à définir la frontière à partir des mouvements migratoires, c'est-à-dire à partir de l'ampleur spatio-temporelle des champs migratoires qu'elle produit. La frontière n'est plus alors seulement le produit, ou plus, la propriété des Etats, elle est aussi le produit des individus qui la parcourent, un champ migratoire et un champ d'actions à partir duquel s'organisent des échanges, des coalitions, des luttes.

La notion de «frontière mobile» (Amilhat-Szary, 2012; AmilhatSzary, Giraut, 2011) est particulièrement intéressante pour désigner à la fois les déplacements de la frontière, les déplacements frontaliers, ainsi que les relations entre ces différentes dimensions dynamiques. Nous avons tenté de montrer que le cinéma, notamment fictionnel, pouvait contribuer aux réflexions géographiques sur la frontière mobile. Les dimensions cartographiques du cinéma, théorisées par les travaux de Tom Conley et de Teresa Castro, autorisent à créer de nombreuses analogies entre la construction des images cinématographiques et des cartes. L'image cinématographique permet aussi de suggérer de nouvelles formes de visualisation et d'analyse de l'espace géographique. Par ailleurs, la fiction cinématographique, en tant que modèle virtuel, peut servir à interroger, critiquer, dénaturaliser les modèles géographiques, sans que l'expérimentation ne soit «directement sanctionnée par le réel » (Schaeffer, 2012).

Ainsi, l'analyse filmique de Sin Nombre et No Country For Old Men a permis d'envisager la frontière depuis les points de vue changeants de personnages en fuite. Les frontières y apparaissent instables, étendues situées entre un monde plus ou moins connu et un monde inconnu. La 
profondeur des étendues frontalières évoluent en fonction des voyages et des événements.

A partir de données qualitatives, recueillies lors de parcours migratoires suivis et à travers les récits migratoires, il est possible d'envisager la construction de cartes animées, où l'espace-temps de la traversée frontalière changerait de dimension au fur et à mesure de la progression du voyage. Les toponymes, évanescents, apparaîtraient plus ou moins longuement en fonction du temps passé dans tel ou tel lieu, puis seraient à nouveau plongés dans le hors-champ.

Ces propositions, par ailleurs déjà mises en œuvre dans de nombreuses cartes animées, pourraient être appliquées à l'étude spécifique des étendues frontalières et associées aux recherches sur les cartes sensibles, pour tenter de figurer également les affects et les perceptions sensibles associés aux traversées. Si le cinéma, a fortiori fictionnel, ne doit pas être réduit à sa portée cognitive, son pouvoir de dénaturalisation du visible et de suggestion d'univers alternatifs, est particulièrement intéressant dans la perspective des projets cognitifs de la géographie.

\section{Bibliographie}

Amilhat-Szary A.-L., 2012, «Murs et barrières de sécurité : pourquoi démarquer les frontières dans un monde dématérialisé ? », in Ghorra-Gobin C. (dir.), Dictionnaire critique de la mondialisation, Paris, Armand Colin, p. 447-451.

Amilhat-Szary A.-L., Giraut F., 2011, «Frontières mobiles/Mobile borders », http://www.pacte.cnrs.fr/IMG/pdf_BRIT_ProgramFinal_5_9.pdf

Arab C., 2007, «La «Hrague », ou comment les Marocains brûlent les frontières », Hommes et Migrations, n¹266, p. 82-94.

Audebert C., Robin N., 2009, « L'externalisation des frontières des « Nordsanbsp;» dans les eaux des « Suds»», Cultures \& Conflits, n73, http://conflits.revues.org/17512

Benjamin W., 2000, « L'œuvre d'art à l'ère de sa reproductibilité technique », trad. R. Rochlitz, in Euvres, tome III, Paris, Gallimard, p. 67-113.

Bennafla K., Peraldi M., 2008, «Introduction. Frontières et logiques de passage : l'ordinaire des transgressions », Cultures \& Conflits, $\mathrm{n}^{\circ} 72$, p. 7-12. 
Besse J.-M., 2001, «Cartographier, construire, inventer. Notes pour une épistémologie de la démarche de projet », Les Carnets du paysage, $\mathrm{n}^{\circ} 7, \mathrm{p}$. 127-145.

Besse J.-M., 2008, «Cartographie et pensée visuelle. Réflexions sur la schématisation graphique ", in Laboulais I. (dir.), Les usages des cartes (XVIIe-XIXe siècle). Pour une approche pragmatique des productions cartographiques, Strasbourg, Presses universitaires de Strasbourg, p. 19-32, http://halshs.archives-ouvertes.fr/halshs-00256710/

Besse J.-M., 2010, « Cartographies », Les Carnets du paysage, n²0, p. 5-9

Brinckeroff J. J., 2003, A la découverte du paysage vernaculaire, ArlesVersailles, Actes Sud-ENSP, 277 p.

Bruns J., 2011, «The map is not the country: cartography in Joel and Ethan's Cohen No Contry For Old Men », Film Criticism, vol. 36, n², http://www.thefreelibrary.com/The map is not the country: cartography in Joel and Ethan Coen's No...-a0277674701

Caquard S., 2009, «Foreshadowing Contemporary Digital Cartography: A Historical Review of Cinematic Maps in Films », The Cartographic Journal, vol. 46, n 1, p. 46-55.

Caquard S., Taylor F., 2009, «What is Cinematic Cartography ?», The Cartographic Journal, vol. 46, n 1 , p. 5-8

Carrillo A., 2008, «Migration de transit au Mexique : gestion politique et expériences d'acteurs », Actes des Journées d'étude Migrations, nouvelles pratiques, approches plurielles, EHESS, http://journeesmigrations.free.fr/wordpress/?page id=9

Castro T., 2008, «Les cartes vues à travers le cinéma », Cartes et Plans, $\mathrm{n}^{\circ} 2$, p. 1-17

Castro T., 2011, La pensée cartographique des images, Lyon, Aléas, 258 p.

Cesari-Stricker F., 2008, Cormac McCarthy: les romans du Sud-Ouest, Paris, Ophrys, 168 p.

Chenet M., Simoes L., Laurent Q., 2011, «Pratique et enseignement de l'audiovisuel en géographie », EchoGéo, n¹8, http://echogeo.revues.org/12734

Cheylan J-P., 2007, « Les processus spatio-temporels: quelques notions et concepts préalables à leur représentation », Mappemonde, $\mathrm{n}^{\circ} 87$, 
http://mappemonde.mgm.fr/num15/articles/art07303.html

Chivallon C., 2008, « L'espace, le réel et l'imaginaire : a-t-on encore besoin de la géographie culturelle ? », Annales de géographie, n 660-661, p. 6789.

Choplin A., Pliez O., 2011, «De la difficulté de cartographier l'espace saharo-sahélien », Mappemonde, $\mathrm{n}^{\circ} 103$, http://mappemonde.mgm.fr/num31/index.html

Conley T., 2007, Cartographic Cinema, Minneapolis, University of Minnesota Press, 336 p.

Dufour E., 2009, Qu'est-ce que le cinéma?, Paris, Vrin, 125 p.

Équipe MOBYDIC, 2010, «Représentations graphiques et indicateurs des mobilités et des dynamiques de peuplement: contribution bibliographique », http://halshs.archives-ouvertes.fr/halshs-00470407/m Green N., 1999, «Trans-frontieres : Pour une analyse des lieux de passage », Socio-anthropologie, $\quad \mathrm{n}^{\circ} 6, \quad \mathrm{http}: / /$ socioanthropologie.revues.org/index $110 . \mathrm{html}$ Gresh A., Rekacewicz P., 2012, «Ne pas reproduire le visible, mais rendre visible », Le Monde Diplomatique, http://www.mondediplomatique.fr/2012/03/GRESH/47522

Kaddouri L., 2008, «Réflexion sur la sémiologie graphique animée des flux », $\quad$ Mappemonde, $\quad \mathrm{n}^{\circ} 89$, http://mappemonde.mgm.fr/num17/articles/art08104.html

Mains S. P., 2004, «Imagining the border and Southern spaces: Cinematic explorations of race and gender », GeoJournal, vol. 59, p. 253-264

Migreurop, Clochard O., 2009, Atlas des migrants en Europe. Géographie critique des politiques migratoires, Paris, Armand Colin, $144 \mathrm{p}$.

Montebello P., 2008, Deleuze, philosophie et cinéma, Paris, Vrin, 134 p.

Olmedo E., 2011, «Cartographie sensible, émotions et imaginaire », Visions Cartographiques, Blog $d u \quad$ Monde Diplomatique, http://blog.mondediplo.net/2011-09-19-Cartographie-sensible-emotions-etimaginaire

Pian A., 2009, Aux nouvelles frontières de l'Europe : L'aventure incertaine des Sénégalais au Maroc, Paris, La Dispute, 237 p.

Pliez O., 2011, Les cités du désert : des villes sahariennes aux saharatowns, 
Marseille-Toulouse, IRD, Presses Universitaires du Mirail, 163 p.

Poisson M., 2010, "Graphie du déplacement », Les Carnets du Paysage, n²0, p. 104-115

Poli R., 2007, «Migrations de footballeurs et mondialisation: du systèmemonde aux réseaux sociaux », Mappemonde, $\mathrm{n}^{\circ} 87$, http://mappemonde.mgm.fr/num16/articles/art07401.html

Retaillé D., 2011, « La transformation des formes de la limite », ArticuloJournal of Urban Research, nº, http://articulo.revues.org/1723

Schaeffer J.-M., 2002, "De l'imagination à la fiction », Vox Poetica, http://www.vox-poetica.org/t/articles/schaeffer.html

Staszak J.-F., 2011, «La fabrique cinématographique de l'altérité Les personnages de «Chinoises » dans le cinéma occidental», Annales de géographie, $\mathrm{n}^{\circ} 682$, p. 577- 603

Tiberghien G. A., 2004, « Hodologique », Les carnets du paysage, $\mathrm{n}^{\circ} 11, \mathrm{p}$. 6-33

Légende des figures :

Figure 1- Vue d'ensemble et point de fuite, arrêt sur image, No Country For Old Men

Figure 2- Vue d'ensemble plongeante et point de fuite, arrêt sur image, Sin Nombre

Figure 3- Carte topographique et hors-champ, arrêt sur image, Sin Nombre

Figure 4- Succession de plans d'ensemble de l'étendue frontalière, arrêts sur image, No Country For Old Men

Figure 5- Le shérif Bell et la carte du Texas, arrêt sur image, No Country For Old Men

Figure 6- La carte illisible, arrêt sur image, No Country For Old Men 